\title{
The Sleep Disturbances in Elderly Patients with Chronic Liver Disease
}

\author{
Hamza SA, Rasha Ahmed Mohamed, Nesma Gamal El Sheikh, and Nourhan Basyouny. \\ Geriatrics \& Gerontology department, Faculty of Medicine, Ain Shams University, Cairo, Egypt.
}

\begin{abstract}
Background: Sleep disturbances complaints are common problems among elderly patients. There is a high prevalence among older adults especially those with chronic liver disease and this greatly affect their quality of life
\end{abstract}

Aim: To study the relationship between chronic liver disease and sleep disturbances among elderly patients

Methods: Case control study included 100 elderly participants, divided equally to cases and controls, cases are further divided into child $A$ and child B. Pittsburgh sleep quality index using Arabic version was used to detect sleep disturbance

Results: The study showed significant relationship between chronic liver disease and sleep disturbances as $29 \%$ of total number of cases did have sleep disturbances while only $14 \%$ of controls did. Moreover, $32 \%$ of child $\mathrm{A}$ group and $56 \%$ among child B group did have sleep disturbances. As regard component of Pittsburg sleep quality index, the study showed that there was significant difference in subjective sleep quality, sleep latency, sleep efficiency, daytime dysfunction.

Conclusions: Sleep disorders prevalence among elderly increases with the presence of chronic liver disease.

Keywords: sleep disturbances, elderly, chronic liver disease, Pittsburgh sleep quality index, Child-Pugh score

\section{Background:}

Sleep disturbances are a major health issue affecting all elderly population and the prevalence continue to increase among elderly with higher prevalence among older adults. For example primary sleep disorders have higher prevalence among older adults. ${ }^{(1)}$

Sleep related disorders among elderly have a multifactorial etiology. This includes primary and secondary causes. Primary causes include sleepdisordered breathing, periodic limb movements in sleep, restless legs syndrome, rapid eye movement (REM), sleep behavior disorder, insomnia, and circadian rhythm disturbances. Secondary causes such as lifestyle and environmental factors, multiple comorbid medical conditions, the use of medication for comorbid and psychiatric conditions. ${ }^{(1)}$

Chronic liver disease is one of the medical comorbidities that are associated with sleeping disturbances especially among elderly and this can be due to several factors such as pain, discomfort from ascites, fatigue associated with the disease, poor sleep hygiene, medications affecting sleep, as well as pruritus (especially in primary biliary cirrhosis ${ }^{\text {)(2), }}$ but also the pathophysiology of liver disease itself represent a direct consequence of sleep dysfunction for example Insomnia is documented in well-compensated cirrhotic patients with no other causes explaining disturbed sleep such as itching, tense ascites, or diuretics treatment ${ }^{(3)}$.

Sleep disturbances among patients with chronic liver disease was first described in 1954 among cirrhotic patients with severe overt hepatic encephalopathy (HE) as it was presented with sleep-wake inversion (the combination of restless nights and excessive daytime sleepiness), latter on with more studies conducted on sleep disturbance they found that an extreme wide range of sleep disturbances can be seen among cirrhotic patients even without signs of overt $\mathrm{HE}$ and these studies documented that the prevalence of sleep difficulties include falling asleep, multiple night awakenings and daytime sleepiness varying from 27 to $70 \%$ among patient with chronic liver disease ${ }^{(4)(5) .}$

Studies found that patients with cirrhosis usually presented with delayed sleep habits/evening preference, 
these features are associated with increase sleep latency and so worse sleep quality ${ }^{(3)}$.

Montagnese et al. explained that one of the causes of disturbed sleep among patient with chronic liver disease is the abnormal interaction between the two primary components that regulate sleep the homeostatic process that builds up during wakefulness and declines during sleep, and the circadian process (6).

Also there are suggestions that there is multiple unexplored pathophysiological mechanisms through which cirrhosis and its complications might affect sleep-wake patterns, as sleep abnormalities have also been reported in patients with little or no evidence of neuropsychiatric disturbance related to $\mathrm{HE}^{(7)}$

In patients with cirrhosis the use of 24-h rhythm of skin temperature and the distal proximal gradient showed impaired thermoregulation, in terms of absolute values and time-course. These alterations may be explained by the hyperdynamic circulatory syndrome, caused by the generalized state of vasodilatation which affects heat dissipation in these patients, these abnormalities reflect the severity of the disease and the associated sleepwake abnormalities $(\mathbf{8})(\mathbf{9})$.

Another method used to confirm the evidence of sleep disturbance among chronic liver disease is glucose fluctuations, as greater fluctuations were associated with higher Pittsburgh sleep quality index scores, studies showed that $70 \%$ of cirrhotic patients suffer from glucose intolerance or diabetes, so it partly explains the disturbed sleep patterns in these patients. (10)

This study was conducted to investigate the relationship between chronic liver disease and sleep disturbances among elderly patients.

\section{Methods}

A case control study was conducted on 100 elderly participants (60 years and older) divided into two groups and they were recruited from outpatient clinics of both the Geriatric department and Tropical department of Ain Shams University hospitals:

Cases included 50 chronic liver disease patients which are further divided into 2 groups according to ChildPugh criteria into child A and child B

Exclusion criteria of cases:

Chronic liver disease patient Child-Pugh criteria child C, history of hepatic encephalopathy, history of any Psychiatric disorder, any end organ failure as heart failure and renal failure.

Control group included 50 healthy non-hepatic elderly individuals. Matched with the cases in age, sex and comorbidities

\section{Study tools}

All participants were subjected to

1. Oral consent that they agree to participate

2. Assessment including detailed history taking and examination.
3. Evaluation of sleeping pattern by Pittsburgh sleep quality test (11)

4. Investigations as INR, liver enzymes, bilirubin level, Albumin, and Pelvi-abdominal ultrasound to confirm the diagnosis of chronic liver disease and to classify the patients according to child score

\section{Statistical analysis}

Analysis of data was performed by using the Statistical Package for Social Science (SPSS 16). Comparison between quantitative variables was done using t-test to compare two groups. Comparison of qualitative variables was done using the Chi-square test or Fisher's exact test when appropriate. Significant level was measured according to $\mathrm{P}$ value (probability), $\mathrm{P}>0.05$ is insignificant, $\mathrm{P}<0.05$ is significant and $\mathrm{p}<0.01$ is highly significant.

\section{Ethical consideration}

Informed oral consent was taken from every patient after explanation of the study aim and procedure and the study methodology was reviewed and approved by the Research Review Board of the Geriatrics and Gerontology Department, Faculty of medicine, Ain Shams University.

\section{Results}

The study was conducted on 100 participants classified into equal number of control and cases and cases were further divided into two groups child A and child B, all participants are 60 years and older and were classified into different age groups, the three groups are statically matched as regard age and gender (Table 1).

The study results showed significant relationship between sleep disturbances and chronic liver disease as $24 \%$ of cases with hepatic disease had sleep disturbances in comparison to only $14 \%$ of controls, comparing the two group of cases together (Child A and Child B ) the results showed that in child A $32 \%$ had sleep disturbances while in child B 56\% of participants complained of sleep disturbances and these difference were statically significant (P-value of 0.001) (Table 2).

Comparing each component of Pittsburgh sleep quality index among cases and control showed a statistically significant relationship between each Pittsburgh sleep quality index component and chronic liver disease (Table 3).

Except for Component $\mathbf{6}$ of Pittsburgh sleep quality index (use of sleep medication) had no statistical difference as none of cases in the child A or child B candidates used sleep medications. (Table 3) 
Table 1: Demographic data of the participants:

\begin{tabular}{|c|c|c|c|c|c|c|c|c|c|}
\hline & & \multicolumn{2}{|l|}{ Control } & \multicolumn{2}{|c|}{ Child A } & \multicolumn{2}{|c|}{ Child B } & \multicolumn{2}{|c|}{ Total } \\
\hline & & $\mathbf{N}$ & $\%$ & $\mathbf{N}$ & $\%$ & $\mathbf{N}$ & $\%$ & $\mathbf{N}$ & $\%$ \\
\hline \multirow[t]{4}{*}{ Age } & 60-65 Years & 15 & 30.00 & 7 & 28.00 & 7 & 28.00 & 29 & 29.00 \\
\hline & 66-70 Years & 15 & 30.00 & 11 & 44.00 & 10 & 40.00 & 36 & 36.00 \\
\hline & 71-75 Years & 12 & 24.00 & 6 & 24.00 & 7 & 28.00 & 25 & 25.00 \\
\hline & 76-80 Years & 8 & 16.00 & 1 & 4.00 & 1 & 4.00 & 10 & 10.00 \\
\hline \multirow[t]{2}{*}{ Gender } & Female & 25 & 50.00 & 12 & 48.00 & 12 & 48.00 & 49 & 49.00 \\
\hline & Male & 25 & 50.00 & 13 & 52.00 & 13 & 52.00 & 51 & 51.00 \\
\hline
\end{tabular}

Table 2: Relationship between Pittsburgh sleep quality index total score and hepatic and non-hepatic patients Participant

\begin{tabular}{|c|c|c|c|c|c|c|c|c|c|c|c|}
\hline \multirow[b]{2}{*}{ Pittsburgh } & & \multicolumn{8}{|c|}{ Participant } & \multicolumn{2}{|c|}{ Chi-Square } \\
\hline & & \multicolumn{2}{|c|}{ Control } & \multicolumn{2}{|c|}{ Child A } & \multicolumn{2}{|c|}{ Child B } & \multicolumn{2}{|c|}{ Total } & & \\
\hline \multirow{3}{*}{ Total Component } & & $\mathbf{N}$ & $\%$ & N & $\%$ & $\mathbf{N}$ & $\%$ & $\mathbf{N}$ & $\%$ & $X^{2}$ & P-value \\
\hline & Less than 5 & 43 & 86 & 17 & 68 & 11 & 44 & 71 & 71 & 14.424 & $0.001 *$ \\
\hline & More than 5 & 7 & 14 & 8 & 32 & 14 & 56 & 29 & 29 & & \\
\hline
\end{tabular}

Table 3: The relationship between Pittsburgh sleep quality index components in hepatic and non-hepatic participants Pittsburgh

Pittsburgh sleep quality index components
Participants
Control

\section{Child A Child B Total}

Component 1

Subjective sleep quality

Component 2

Sleep latency

Component 3

Sleep duration

Component 4

Sleep efficiency

Component 5

Sleep disturbances

Component 6

Use of sleep Medication

Component 7

Daytime dysfunction

$\begin{array}{lllllllllll} & \mathrm{N} & \% & \mathrm{~N} & \% & \mathrm{~N} & \% & \mathrm{~N} & \% & \mathrm{X} & \text { P-value } \\ \text { No difficulty } & 40 & 80 & 0 & 0 & 0 & 0 & 40 & 40 & 98.333 & <0.001^{*} \\ \text { Mild difficulty } & 10 & 20 & 25 & 100 & 13 & 52 & 48 & 48 & & \\ \text { Moderate } & 0 & 0 & 0 & 0 & 12 & 48 & 12 & 12 & & <0.001^{*} \\ \text { No difficulty } & 30 & 60 & 0 & 0 & 0 & 0 & 30 & 30 & 54.240 & \\ \text { Mild difficulty } & 20 & 40 & 17 & 68 & 13 & 52 & 50 & 50 & & \\ \text { Moderate } & 0 & 0 & 8 & 32 & 12 & 48 & 20 & 20 & & \\ \text { No difficulty } & 26 & 52 & 17 & 68 & 0 & 0 & 43 & 43 & 30.428 & <0.001^{*} \\ \text { Mild difficulty } & 16 & 32 & 8 & 32 & 15 & 60 & 39 & 39 & & \\ \text { Moderate } & 8 & 16 & 0 & 0 & 10 & 40 & 18 & 18 & & <0.001^{*} \\ \text { No difficulty } & 50 & 100 & 0 & 0 & 9 & 36 & 59 & 59 & 93.066 & \\ \text { Mild difficulty } & 0 & 0 & 18 & 72 & 5 & 20 & 23 & 23 & & \\ \text { Moderate } & 0 & 0 & 7 & 28 & 8 & 32 & 15 & 15 & & \\ \text { Severe } & 0 & 0 & 0 & 0 & 3 & 12 & 3 & 3 & & \\ \text { No difficulty } & 2 & 4 & 0 & 0 & 0 & 0 & 2 & 2 & 20.988 & \\ \text { Mild difficulty } & 48 & 96 & 18 & 72 & 16 & 64 & 82 & 82 & & \\ \text { Moderate } & 0 & 0 & 7 & 28 & 9 & 36 & 16 & 16 & & \\ \text { No difficulty } & 46 & 92 & 25 & 100 & 25 & 100 & 96 & 96 & 4.167 & 0.125 \\ \text { Mild difficulty } & 4 & 8 & 0 & 0 & 0 & 0 & 4 & 4 & & <0.001^{*} \\ \text { No difficulty } & 20 & 40 & 0 & 0 & 1 & 4 & 21 & 21 & 61.023 & \\ \text { Mild difficulty } & 28 & 56 & 18 & 72 & 11 & 44 & 57 & 57 & & \\ \text { Moderate } & 2 & 4 & 7 & 28 & 2 & 8 & 11 & 11 & & \\ \text { Severe } & 0 & 0 & 0 & 0 & 11 & 44 & 11 & 11 & & \end{array}$




\section{Discussion}

Sleep disturbance affect function and daytime activity of elderly thus affect quality of life, so special attention should be given to recognize causes of sleep disturbance among elderly with chronic liver disease, and to identify which part of sleep is affected so a management plan could be done to improve the sleep quality of elderly.

This study included 100 male and female elderly, with ages ranging from 60-80 divided into cases and controls and cases are then divided into Child A and Child B.

Studies concerned with sleep disturbances and chronic liver disease was not conducted before on Egyptian elderly and this study intended to investigate the effect of heterogenecity as regards nationality, race and ethnicity.

our study showed a statistical significance between sleep disturbances and chronic liver disease as assessed by Arabic version of Pittsburgh sleep quality index , we concluded that sleep disturbance is more frequent among participants with chronic liver disease than the control (24\% of cases with hepatic disease had sleep disturbances in comparison to only $14 \%$ of controls)

Moreover, sleep disturbances increase with increased severity of chronic liver disease. when comparing the two groups of cases together (Child A and Child B ) the results showed that $32 \%$ of child A patients had sleep disturbances while in child B $56 \%$ of participants complained of sleep disturbances and these differences were statistically significant.

this agrees with other studies as Montagnese S, et al., $2009^{(3)}$, Marques DM, et al., $2016^{(13}$ )and Tsai CF et a., 2016 ${ }^{(\mathbf{1 2}}$ ) all showed that cirrhotic patients were identified as 'poor' sleepers (PSQI > 5).

Our study revealed that the most affected component of Pittsburgh sleep quality index in comparison to control individual are sleep quality, sleep latency, sleep efficiency and daytime dysfunction, moreover, the sleep disturbances increased with the increased severity of hepatic condition.

Similar findings have been obtained by AL-Jahdali $\mathbf{H}$ et al., $2014^{(15)}$ and Bruyneel $M$ et al., $2018{ }^{(14)}$ their results showed that there was a significant relationship between severity of liver cirrhosis and prevalence of sleep disturbances when compared to healthy subjects.

When studying the relation between sleep medications use (component 6 in PSQI) in different study groups there was no significant relation among groups that may be due to fear of use sleep medications in elderly hepatic patients
This agrees with a study made by Franz CC et al., 2012 $^{(16)}$ which showed that Antidepressants, benzodiazepines and other psychotropic and central nervous system agent should be used with caution or avoided in chronic liver disease patients.

\section{Conclusion}

Sleep disorders percentage among elderly increases with the presence of chronic liver disease patients and this significantly causes them to have daytime dysfunction.

The most affected components Subjective sleep quality, sleep latency, sleep efficiency and daytime dysfunction are common complaints among chronic liver disease elderly patients.

Percentage of sleep disturbances increase with severity of chronic liver disease

\section{References}

1. Susan K. Roepke \& Sonia Ancoli-Israel Sleep disorders in the elderly, Indian J Med Res, February 2010, pp 302-310.

2. Montagnese S, Nsemi LM, Cazzagon N, et al. Sleep-wake profiles in patients with primary biliary cirrhosis. Liver Int. 2013;33(2):203-

3. Montagnese S, Middleton B, Skene DJ and Morgan MY. Night-time sleep disturbance does not correlate with neuropsychiatric impairment in patients with cirrhosis. Liver Int. 2009;29(9):13721382.

4. De Cruz S, Espiritu J, Zeidler M and Wang T. Sleep disorders in chronic liver disease. Seminars in Respiratory and Critical Care Medicine. 2012:33(1):26-35.

5. Bianchi G, Marchesini G, Nicolino F,et al. Psychological status and depression in patients with liver cirrhosis. Dig Liver Dis. 2005;37(8):593-600.

6. Borbély AA. A two process model of sleep regulation. Hum Neurobiol. 1982;1(3):195-204.

7. Mostacci B, Ferlisi M, Baldi Antognini A,et al. Sleep disturbance and daytime sleepiness in patients with cirrhosis: a case control study. Neurol Sci. 2008:29(4):237-40.

8. Garrido M, Saccardo D, De Rui M, et al. Abnormalities in the 24hour rhythm of skin temperature in cirrhosis: sleep-wake and general clinical implications. Liver Int. 2017:37(12):1833-42.

9. Bolognesi M, Di Pascoli M, Verardo A and Gatta A. Splanchnic vasodilation and hyperdynamic circulatory syndrome in cirrhosis. World J Gastroenterol. 2014;20(10):2555-2563.

10. Haraguchi M, Miyaaki $\mathrm{H}$, Ichikawa $\mathrm{T}$, et al. Glucose fluctuations reduce quality of sleep and of life in patients with liver cirrhosis Hepatol Int. 2017;11(1):125-131.

11. Daniel J.Buysse and Charles F.ReynoldsIII, The Pittsburgh sleep quality index: A new instrument for psychiatric practice and research,May 1989.

12. Tsai CF, Chu CJ,Wang Y, et al. Increased serum interleukin-6, not minimal hepatic encephalopathy, predicts poor sleep quality in nonalcoholic cirrhotic patients.Aliment Pharmacol Ther. 2016 Oct;44(8):836-45.

13. Marques DM, Teixeira HR Lopes AR, et al. Sleep Quality Assessment and Daytime Sleepiness of Liver Transplantation Candidates. Transplant Proc. 2016;48(7):2356-2360

14. Bruyneel M and Sersté T,Sleep disturbances in patients with liver cirrhosis: prevalence, impact, and management challenges ,Nat Sci Sleep. 2018; 10: 369-375

15. AL-Jahdali H, Al Enezi A, Anwar A, et al 2014 Prevalence of Insomnia and Sleep Patterns among Liver Cirrhosis Patients. Journal of Circadian Rhythms,12(1): 2, pp. 1-6

16. Franz CC, Egger S, Born C, et al. Potential drug-drug interactions and adverse drug reactions in patients with liver cirrhosis. Eur $\mathrm{J}$ Clin Pharmacol 2012; 68: 179-88. 